\title{
Use of Triamcinolone to Reduce the Inflammation Associated with Kybella Injection
}

\author{
Mojgan Hosseinipour ${ }^{1}$, Nwanneka Okwundu ${ }^{2 *}$, Monica Huynh ${ }^{1}$, Sonya Zarkhin ${ }^{1}$, Dimitria Papadopoulos ${ }^{1}$, \\ Cindy Hoffman ${ }^{1}$
}

${ }^{1}$ St Barnabas Hospital Dermatology Residency Program, Bronx, New York, USA

${ }^{2}$ Department of Dermatology, University of Utah Salt-lake City, Utah, USA

Received: February 10, 2020; Accepted: February 21, 2020; Published: March 16, 2020

*Corresponding author: Nwanneka Okwundu,University of Utah Department of Dermatology, Salt-lake City, Utah, USA. E-mail: pishgouym951@ mums.ac.ir

\begin{abstract}
Background: Submental fullness has been a concern for patients in dermatology for years. Liposuction and cosmetic surgery were considered the mainstay for elimination of unwanted submental fat. However, these procedures are invasive and associated with major complications like prolonged recovery times, and the risk of contour irregularities. In 2015, Kybella (deoxycholic acid) was approved as a first-in-class injectable agent for the treatment of submental fullness. Although, it is non-invasive and has less complications than plastic surgery, it is still associated with adverse effects such as pain, swelling/edema, and bruising. The most concerning adverse effect for patients is the prolonged inflammation that may occur for up to 2 weeks post-injection.
\end{abstract}

Objective: This is a comparison trial of deoxycholic acid injections with or without triamcinolone acetate for the reduction of submental fat in one patient. The study will also assess the effect of triamcinolone acetate on post-injection inflammation associated with deoxycholic acid injection in the treatment of submental fullness.

Method: A 35-year-old female presented for evaluation of submental fullness and opted for treatment with Kybella. Her right submental treatment area was injected with $2 \mathrm{ml}$ of deoxycholic acid $10 \mathrm{mg} / \mathrm{ml}$ plus $0.2 \mathrm{ml}$ triamcinolone acetate. The left submental treatment area was treated with $2 \mathrm{ml}$ of deoxycholic acid $10 \mathrm{mg} /$ $\mathrm{ml}$ alone. The patient was observed over a duration of 3 months to evaluate inflammation and treatment response between the two treatment zones.

Results: This split-neck study resulted in decreased postinjection swelling. However, at 3-month follow-up, there was no clinical improvement to the submental fullness bilaterally.

Limitations: Small sample size, lack of objective measures, possible diffusion between treatment zones, and prior deoxycholic acid treatment.

Conclusion: Triamcinolone can decrease post-injection swelling if given as an adjunct to Kybella treatment.

Keywords: Kybella; Submental; Triamcinolone; Deoxycholic Acid; Inflammation

\section{Introduction}

The chin and the neck have a significant contribution to the aesthetics of the face, hence any unconventional appearance of its features such as excess fat, may be bothersome to the patients [1-3]. According to a survey conducted by the American Society for Dermatologic Surgery (ASDS), 67\% of the 7,315 respondents reported being "somewhat to extremely bothered" by excess fat under the chin or neck [4]. Over the years reduction of submental fat has been achieved with invasive surgical procedures such as liposuction, direct fat excision, and/or face/neck lift. However, due to the complications associated with invasive surgeries and their long recovery times, they are not a viable option for some patient populations [5-7].

A few non-invasive methods have been developed for the reduction of submental fullness. Noninvasive energy devices such as ultrasound or controlled cooling have been used for targeted destruction of subcutaneous fat cells. However, the techniques have not been rigorously tested and are not all currently optimized for submental contouring. The results have been variable and debatable as well [4]. In 2015, a new noninvasive method of submental fat reduction called Kybella was approved by the Food and Drug Administration (FDA). This drug has gone through decades of development and rigorous clinical trials. Kybella (Kythera Biopharmaceuticals, Westlake Village, California) is a first-in-class injectable agent approved for the reduction of submental fat $[8,9]$. It is a proprietary formulation of synthetic (nonanimal, nonhuman) deoxycholate that has gone through series of trials including 18-Phase 1 to 3 clinical studies to support the current indication, efficacy and safety. The significant improvement observed has also been correlated with objective improvement on magnetic resonance imaging $[10,11]$.

Though Kybella treatment is non-invasive and its safety has been thoroughly tested, it is not without side effects. The most common adverse effects of Kybella are associated with the injection-sites. These adverse effects occur in at least $20 \%$ of patients and at a higher rate than with placebo. The treatment related adverse effects include edema, bruising, pain, numbness, erythema, and induration at the injection site, which sometimes lasts for more than 30 days. Marginal mandibular nerve injury (asymmetric smile, facial muscle weakness) occurred in $4 \%$ and dysphagia occurred in $2 \%$ of patients treated with the drug. All but one case resolved without treatment $[7,12,13]$. Different 
methods have been employed to alleviate these adverse effects including ice, NSAIDs, topical or injectable amides anesthetics, vibration, and botulinum toxin injection for pain reduction $[4,14,15]$. A small study showed that transdermal treatment with corticosteroids immediately after hyaluronic acid injections reduced swelling[16]. Hence, we utilized triamcinolone to alleviate the inflammatory effects of the Kybella injection and observed for changes in inflammatory response and patient outcome.

\section{Methods}

A 35-year-old female with a history of one previous treatment with Kybella greater than one year prior to office visit presented for evaluation of submental fullness. She elected for treatment of her submental fullness with deoxycholic acid. The intensity and laxity of submental fullness was evaluated by pinching and pulling. The patient was also asked to smile, swallow, and grimace to evaluate the platysma. The injection area was cleaned. Her submental treatment area marked for treatment was bounded superiorly by a line $1 \mathrm{~cm}$ inferior to the mandibular margin, laterally by the sternocleidomastoid muscles, and inferiorly by the hyoid bone and divided into right and left portions. Transfer paper with printed grid pattern was pressed on the side of her skin. Cotton ball soaked with sterile water was pressed firmly on it and the paper was peeled.

In the right submental treatment area, $2 \mathrm{ml}$ of deoxycholic acid $10 \mathrm{mg} / \mathrm{ml}$ plus $0.2 \mathrm{ml}$ triamcinolone acetate $10 \mathrm{mg} / \mathrm{ml}$ was mixed and then delivered in 13 injections spaced $1.0 \mathrm{~cm}$ apart at $0.2 \mathrm{~mL} /$ injection using a 30 gauge 0.5 -inch needle. In the left submental treatment area, deoxycholic acid alone $20 \mathrm{mg} / 2 \mathrm{ml}$ was delivered in 13 injections spaced $1.0 \mathrm{~cm}$ apart at $0.2 \mathrm{~mL} /$ injection. At the end, the grid marks were removed with alcohol. The patient was asked to swallow again to re-evaluate submental fullness. The patient was observed over duration of 3 months to evaluate inflammation and treatment response between the two treatment zones. The patient was brought in for re-evaluation at 4,8 , and 12 weeks.

The split-neck study resulted in decreased post-injection swelling. At 12-week follow-up, there was no clinical improvement to the submental fullness bilaterally. The patient did not show or report any side effect associated with topical triamcinolone use during the duration of observation(Figure1-4).

\section{Discussion}

ATX-101 or deoxycholic acid injection (known as Kybella in the United States and Belkyra in Canada) was approved in 2015 as a first-in-class injectable drug for improvement in the appearance of moderate to severe convexity or fullness associated with submental fat $[8,11]$. The idea that led to the development of ATX101 as a pharmacologic treatment for reduction of submental fat was identified by Rotunda and colleagues $[11,17]$.

Deoxycholic acid is the primary mediator of adipocyte lysis in compounded phosphatidylcholine/sodium deoxycholate. When injected into submental fat, ATX-101 physically disrupts

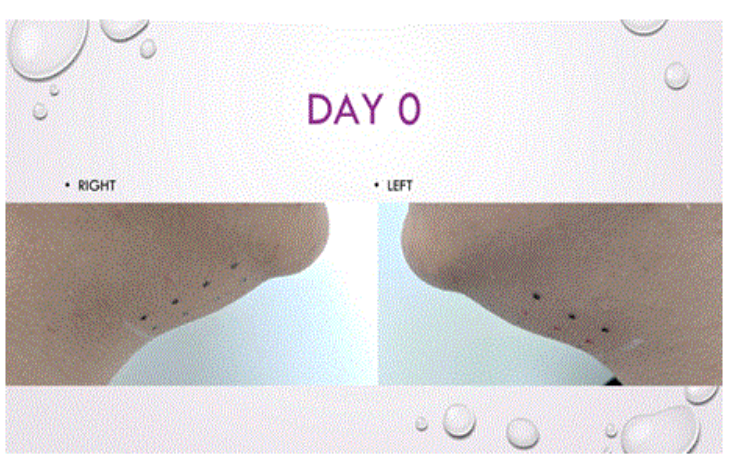

Figure 1: Day 0: Decreased post-injection swelling observed.

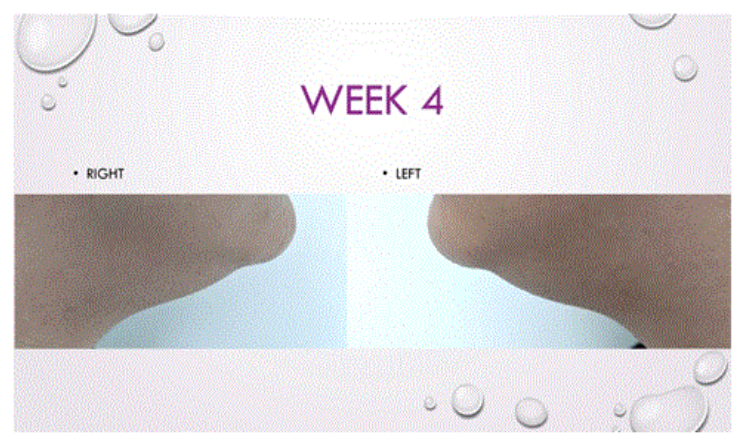

Figure 2: Week 4: No clinical improvement to the submental fullness bilaterally. No side effects of triamcinolone injection observed.

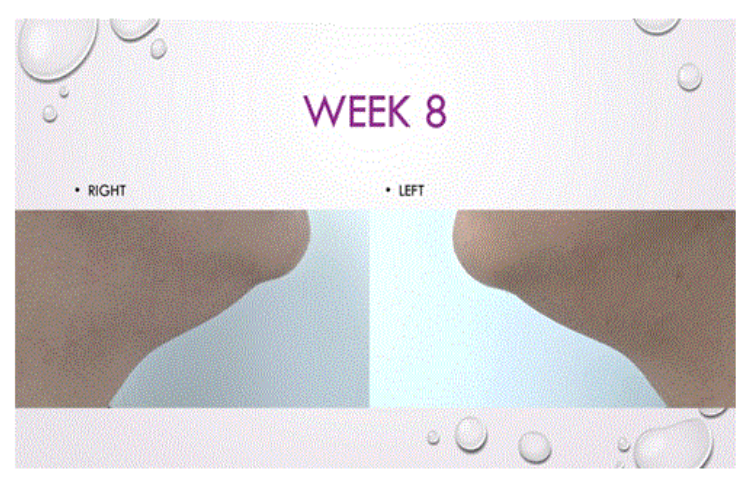

Figure 3: Week 8: No clinical improvement to the submental fullness bilaterally. No side effects of triamcinolone injection observed.

the adipocyte cell membranes and causes cell death as early as 1 day after injection. Subsequently neutrophils invade tissue (day 3), followed by macrophages (day 7), followed by fibroblasts (day 28). By one month, inflammation largely resolves, providing the rationale for the approved monthly intervals between each treatment. Relatively protein-poor tissue such as fat is more sensitive to the cytolytic effects of deoxycholic acid than relatively protein-rich tissues such as skin and muscle $[18,19]$. Due to these properties, Kybella also has an off-label use to correct central abdominal fat, the "muffin top" or "love handle" abdominal fat overhang, lipomas, buttock fat, thigh fat, upper-arm fat, cellulite, and infraorbital fat pads. 


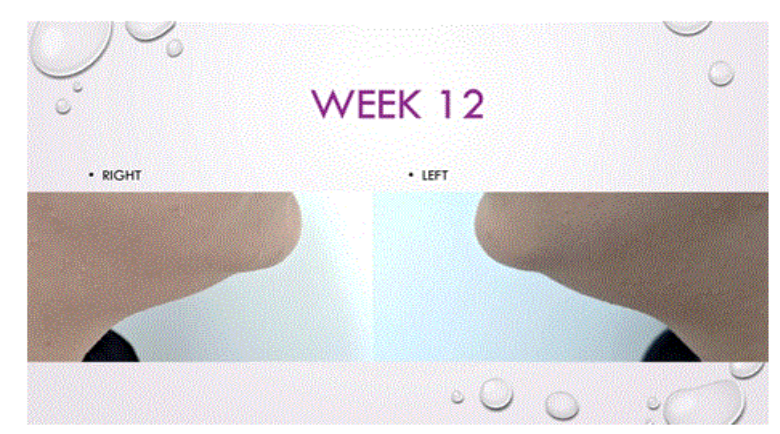

Figure 4: Week 12: No clinical improvement to the submental fullness bilaterally

Kybella has not been found to affect the internal homeostasis of the body. ATX-101 is rapidly absorbed when injected subcutaneously into either submental fat or abdominal fat in healthy subjects. Phase I studies have shown that when ATX-101 is subcutaneously injected into fat, plasma levels of deoxycholic acid rapidly increases, remains within the physiologic range for endogenous deoxycholic acid, and then returns to baseline levels within 12 to 24 hours [20,21]. In addition, these Phase 1 studies have shown that ATX-101 has no clinically meaningful effect on heart rate, QT/QTc intervals on electrocardiography, or plasma concentrations of total cholesterol, triglycerides, lowdensity lipoprotein, high-density lipoprotein, free fatty acids, or proinflammatory cytokines [21]. Rather, fluctuations observed in total cholesterol, triglyceride, and free fatty acid levels after ATX-101 administration were short lived are similar to those observed after ingestion of a meal. Furthermore, all liver and renal function laboratory values were found to be unremarkable [11]. Although, Kybella injections may not alter the internal homeostasis of the body, they are associated with injection site adverse effects. In a study conducted by the United States Food and Drug Administration (FDA), the patients receiving the active drug had the following side effects: edema/swelling (87\%), hematoma/bruising (72\%), pain (70\%), numbness $(66 \%)$, erythema $(27 \%)$, induration $(23 \%)$, and temporary marginal mandibular nerve dysfunction resulting in an asymmetric smile (4\%). In the placebo group of this study, the only comparable adverse event to the active drug Kybella was bruising at 70\% $[9,11,22]$. Fortunately, the injection-site reactions observed in these clinical trials are mostly mild or moderate and transient[22]. Furthermore, the incidence and severity of these adverse effects were also found to decrease after the first treatment $[10,23]$. It was also observed that in ATX-101-treated subjects, peak pain occurred within 1 to 5 minutes of treatment. Most patients reported mild and substantially reduced pain by 15 minutes [24]. Consequently, some studies have attempted different methods of alleviating these adverse effects. In a double-blind, parallel-group, 2-factor, single treatment, exploratory phase $3 \mathrm{~b}$ study, subjects were randomized to 1 of 4 patient experience management paradigms (cold; cold+ anesthetic +epinephrine; cold+ anesthetic +epinephrine+ Antihistamine + NSAID; and cold+ anesthetic +epinephrine+ Antihistamine + NSAID+ chin strap). It was observed that adding topical/injectable lidocaine with epinephrine to ice improves pain by only $17 \%$, whereas the combination of ice, topical/injectable lidocaine with epinephrine, and oral ibuprofen and loratidine reduced peak pain by $40 \%$ compared with cold treatment alone. It was discovered that none of the mitigation paradigms reduced swelling/edema and that epinephrine may reduce bruising. After a single ATX-101 treatment session, $30 \%$ of subjects were considered responders, and $64 \%$ were satisfied with the appearance of their face and chin strap [24].

A literature review of the management of adverse effects associated with ATX-101 treatment in clinical practice was performed. Based on its results, the authors put forward some management considerations with some common clinical interventions. They advise that pretreatment with oral ibuprofen and/or acetaminophen should be done an hour before treatment. In addition, pre-injection with epinephrine containing buffered lidocaine 15 minutes before treatment may help with management of pain and bruising. Alternatively, if local anesthetic pre-injection was not performed, cold application to the treated area before and immediately after the procedure may help to reduce pain and swelling. They also propose discontinuing medications/supplements that cause increased anticoagulant or antiplatelet activity 7 to 10 days before ATX-101 treatment, when possible as this can reduce the risk of bruising $[4,24]$.

Based on the result of our study, we recommend that physicians should consider using triamcinolone as an adjunct in Kybella treatment in addition to the above recommendations. Our study showed decreased post-injection swelling even though there was no clinical improvement to the submental fullness bilaterally at her 3 months follow-up visit. Some limiting factors in this study that may have impacted the result of the 3 months follow-up visit include small sample size, lack of objective measures, possible diffusion between treatment zones, and prior deoxycholic acid treatment [25]. The patient had one previous treatment with Kybella greater than one year prior to her second treatment. This may or may not have affected her outcome. Typically, 2 to 3 treatments of Kybella are recommended for successful treatment of submental fullness, spaced one month apart. Future studies are needed to assess the use of triamcinolone as an adjunct to Kybella in treatment of submental fullness in a larger scale.

\section{References}

1. Raveendran SS, Anthony DJ, Ion L. An anatomic basis for volumetric evaluation of the neck. AesthetSurg J. 2012;32(6):685-691.

2. Honigman R, Castle DJ. Aging and cosmetic enhancement. ClinInterv Aging. 2006;1(2):115-119.

3. Huettner F, Vasconez LO, Torre JI. Neck rejuvenation-anatomy and clinical correlation. Facial Plast Surg. 2012;28(1):40-51.

4. Fagien S, Mcchesney P, Subramanian M, Jones DH. Prevention and Management of Injection-Related Adverse Effects in Facial Aesthetics. Dermatologic Surgery. 2016; 42(Suppl 1):S300-S304. doi: $10.1097 /$ dss.0000000000000898

5. Rotunda AM. Injectable treatments for adipose tissue: terminology, mechanism, and tissue interaction. Lasers Surg 
Med. 2009;41(10):714-720.

6. Wollina U, Goldman A. ATX-101 for reduction of submental fat. Expert OpinPharmacother. 2015;16(5):755-762.

7. Georgesen C, Lipner SR. The development, evidence, and current use of ATX-101 for the treatment of submental fat. Journal of Cosmetic Dermatology. 2017;16(2):174-179. doi: 10.1111/jocd.12347

8. KytheraBiopharmaceuticals. Inc. KYBELLA (deoxycholic acid) injection [prescribing information]. Accessed August 2016.

9. Cohen J. Additional thoughts on the new treatment Kybella. Seminars in Cutaneous Medicine and Surgery. 2015;34(3):138-139. doi: 10.12788/j.sder.2015.0175

10. Jones DH, Carruthers J, Joseph JH, Callender VD, Patricia Walker, Daniel RL, et al. REFINE-1, a multicenter, randomized, double-blind, placebo-controlled, phase 3 trial with ATX-101, an injectable drug for submental fat reduction. Dermatol Surg. 2016;42(1):38-49.

11. Dayan SH, Jones DH, Carruthers J, Humphrey S, Brandt F, Walker P, et al. A pooled analysis of the safety and efficacy results of the multicenter, double-blind, randomized, placebo-controlled phase 3 REFINE-1 and REFINE-2 trials of ATX-101, a submental contouring injectable drug for the reduction of submental fat. PlastReconstr Surg. 2014;134(4S1):123

12. Deoxycholic acid (Kybella) for double chin. Med Lett Drugs Ther. 2015;57(1483):165-166.

13. Mess SA. Lower Face Rejuvenation with Injections. Plastic and Reconstructive Surgery - Global Open. 2017;5(11):e1551.doi: 10.1097/gox.0000000000001551

14. Mally P, Czyz CN, Chan NJ, Wulc AE. Vibration anesthesia for the reduction of pain with facial dermal filler injections. AesthetPlast Surg 2014;38(2):413-418.

15. Sharma P, Czyz CN, Wulc AE. Investigating the efficacy of vibration anesthesia to reduce pain from cosmetic botulinum toxin injections.
AesthetSurg J. 2011;31(8):966-971.

16. Iannitti T, Rottigni V, Palmieri B. Corticosteroid transdermal delivery to target swelling, edema and inflammation following facial rejuvenation procedures. Drug Des DevelTher. 2013;7:1035-1041.

17. Rotunda AM, Suzuki H, Moy RL, Kolodney MS. Detergent effects of sodium deoxycholate are a major feature of an injectable phosphatidylcholine formulation used for localized fat dissolution. Dermatol Surg. 2004; 30(7):1001-1008.

18. Rotunda AM,Ablon G, Kolodney MS. Lipomas treated with subcutaneous deoxycholate injections. J Am AcadDermatol. 2005;53(6):973-978.

19. Thuangtong R, Bentow JJ, Knopp K, Mahmood NA, David NE, Kolodney MS, et al. Tissue-selective effects of injected deoxycholate. Dermatol Surg. 2010;36(6):899-908.

20. Walker P, Fellmann J, Lizzul PF. A phase I safety and pharmacokinetic study of ATX-101: injectable, synthetic deoxycholic acid submental contouring. J Drugs Dermatol. 2015;14(3):279-287.

21. Walker P, Lee D. A phase I pharmacokinetic study of ATX-101: serum lipids and adipokines following synthetic deoxycholic acid injections. J CosmetDermatol. 2015;14(1):33-39.

22. Kybella [package insert]. Westlake, California: Kythera Biopharmaceuticals. 2015

23. Humphrey S, Sykes J, Kantor J, Bertucci V, Walker P, Lee DR, et al. ATX101 for reduction of submental fat: a phase III randomized controlled trial. J Am AcadDermatol. 2016;75(4):788-797.

24. Dover JS, Kenkel JM, Carruthers A, Lizzul PF, Gross TM, Subramanian M, et al. Management of Patient Experience with ATX-101 (Deoxycholic Acid Injection) for Reduction of Submental Fat. Dermatologic Surgery. 2017;43(7):1000. doi:10.1097/dss.0000000000001234

25. Karen JK, Hale EK, Geronemus RG. A simple solution to the common problem of ecchymosis. Arch Dermatol. 2010;146(1):94-95. 